\title{
New evolved planetary nebulae in the southern hemisphere
}

\author{
F. Kerber ${ }^{1}$, M. Roth ${ }^{2}$, A. Manchado ${ }^{3}$, and H. Gröbner ${ }^{1}$ \\ ${ }^{1}$ Institut für Astronomie der Leopold-Franzens-Universität Innsbruck, Technikerstraße 25, A-6020 Innsbruck, Austria \\ ${ }^{2}$ Las Campanas Observatory, Carnegie Institution of Washington, Casilla 601, Chile \\ ${ }^{3}$ Instituto de Astrofisica de Canarias, E-38200, La Laguna (Tenerife), Spain
}

Received October 20, 1997; accepted January 30, 1998

\begin{abstract}
The major purpose of this paper is to investigate the nature of planetary nebulae $(\mathrm{PNe})$ candidates in the southern hemisphere, taken from an internal list. We present spectroscopic observations and imaging of six $\mathrm{PNe}$ identified for the first time. Another candidate turned out to be a galaxy. All observed PNe represent evolved stages, their angular diameter ranging from $15^{\prime \prime}$ to $120^{\prime \prime}$, and exhibit very low surface brightnesses. For three PNe indications of interaction with the interstellar medium (ISM) has been discovered.
\end{abstract}

Key words: surveys — planetary nebulae: general

\section{Introduction}

With this work we present six new planetary nebulae $(\mathrm{PNe})$ in the southern hemisphere. The candidates have been taken from an internal list and complement $12 \mathrm{PNe}$ from the northern hemisphere published recently (Kerber et al. 1996, hereafter Paper I). The objects were discovered by scanning the ESO/SERC survey plates for extended nebulae of low surface brightness, see e.g. Melmer \& Weinberger (1990) for details of the search method; the area covered by the search represents about $40 \%$ of the southern sky.

The importance of the late stages of PN evolution and the interesting finds made over recent years have been described in Paper I; we will not repeat this here. Instead we will concentrate on one aspect, briefly mentioned in Paper I that seems to be highly relevant for some of the objects presented here. The process in point is the interaction of old evolved $\mathrm{PNe}$ with the surrounding ambient interstellar medium (ISM). Although first studied 20 years ago (Smith 1976) it remained somewhat of a curiosity, seemingly applicable only to a few peculiar objects like

$\overline{\text { Send offprint requests to }}$ F. Kerber, florian.kerber@uibk.ac.at e.g. A 35 (Jacoby 1981; Hollis et al. 1996). Only with the work of Borkowski et al. (1990) it became obvious that such an interaction is of importance for many old, evolved objects. Since then a number of examples ranging from the mild e.g. A 34 (Tweedy \& Kwitter 1994) to the extreme of Sh 2-174 (Tweedy \& Napiwotzki 1994) have been identified. Most recently Xilouris et al. (1996) have considerably enlarged the sample of known examples.

The objects presented in this paper nicely demonstrate first that many more galactic PNe can be discovered and second that, as a consequence of the high degree of individuality that is inherent to PNe, every single object is of interest and well worth studying.

The objects discussed here are newly discovered PNe. They are included in the first supplement to the Strasbourg-ESO-Catalogue, by Acker et al. (1996), as private communication. With the spectra and images presented in this article we are able to discuss these nebulae on a more solid basis.

\section{Observations}

The spectra have been obtained in April 1996 with the 2.5-m duPont Telescope at Las Campanas Observatory, Chile. Operating the modular spectrograph we covered the spectral range from $4800 \AA$ to $6800 \AA$ for all objects. Using the TEK 1 CCD detector we obtained a spectral resolution of $2 \AA$ /pixel. The exposure time ranged from 600 to $1800 \mathrm{~s}$. The raw data were calibrated using the standard stars LTT 3218 and 3864 from the lists of Hamuy et al. (1992 and 1994). The resulting spectra are presented in Figs. 1 to 6. Additionally, narrow band images have been obtained for KeWe 5 at ESO's $1.54 \mathrm{~m}$ telescope and for KeWe 2 and NeVe 3-2 with LCO's 2.5-m duPont. The bandwidths were $62 \AA$ for $\left(\mathrm{H}_{\alpha}+[\mathrm{N} \mathrm{II}]\right), 35 \AA$ for $[\mathrm{N} \mathrm{II}]$ and $57 \AA$ for [O III].

The objects observed have all been discovered on ESO/SERC film copies during dedicated searches for PNe by visual inspection, for details of this method see Paper I. 
Table 1. Basic data for the PNe investigated

\begin{tabular}{cclcccccc}
\hline Name & Designation & RA $(2000.0)$ & DEC $(2000.0)$ & $\varnothing\left[^{\prime \prime}\right]$ & Plate & $x[\mathrm{~mm}]$ & $y[\mathrm{~mm}]$ & Remarks \\
\hline KeWe 2 & PN G228.5-11.4 & $06^{\mathrm{h}} 37^{\mathrm{m}} 39.3^{\mathrm{s}}$ & $-18^{\circ} 57^{\prime} 30^{\prime \prime}$ & 30 & ESO 557 & 193 & 206 & \\
KeWe 3 & PN G238.4-01.8 & $07^{\mathrm{h}} 33^{\mathrm{m}} 25.9^{\mathrm{s}}$ & $-23^{\circ} 26^{\prime} 08^{\prime \prime}$ & 120 & POSS 656 & 95 & 220 & also on ESO 492 \\
KeWe 4 & PN G257.8-05.4 & $08^{\mathrm{h}} 05^{\mathrm{m}} 33.7^{\mathrm{s}}$ & $-41^{\circ} 56^{\prime} 31^{\prime \prime}$ & 45 & ESO 312 & 273 & 76 & CS $m_{\mathrm{b}} \approx 21$ \\
NeVe 3-1 & PN G275.9-01.0 & $09^{\mathrm{h}} 34^{\mathrm{m}} 04.6^{\mathrm{s}}$ & $-53^{\circ} 12^{\prime} 24^{\prime \prime}$ & 40 & ESO 166 & 51 & 245 & NeVe GN09.32.3 \\
NeVe 3-2 & PN G326.4+07.0 & $15^{\mathrm{h}} 19^{\mathrm{m}} 43.3^{\mathrm{s}}$ & $-48^{\circ} 59^{\prime} 51^{\prime \prime}$ & 30 & ESO 224 & 269 & 196 & NeVe GN15.16.2 \\
KeWe 5 & PN G348.9+04.6 & $16^{\mathrm{h}} 57^{\mathrm{m}} 57.0^{\mathrm{s}}$ & $-35^{\circ} 24^{\prime} 52^{\prime \prime}$ & 15 & ESO 391 & 71 & 126 & CS $m_{\mathrm{b}} \approx 19.5$ \\
\hline & G272.6-05.3 & $08^{\mathrm{h}} 57^{\mathrm{m}} 54.4^{\mathrm{s}}$ & $-53^{\circ} 52^{\prime} 18^{\prime \prime}$ & 35 & ESO 165 & 78 & 213 & ESO G165-08 \\
\hline
\end{tabular}

Table 1 summarizes some basic data of the PNe. In the first column we give the common name (NeVe 3 stands for Neckel \& Vehrenberg 1990), in the second the designation following the IAU recommendations outlined in the Strasbourg-ESO Catalogue of Planetary Nebulae (Acker et al. 1992). Columns 3 and 4 give equatorial coordinates, measured from the ESO/SERC films given in Col. 6, accurate to about 6 arcsec. The coordinates have been measured using a high resolution digitizer and software developed at the institute. In Col. 5 a diameter is given as measured on the ESO-R film. Columns 7 and 8 give $x$ and $y$ positions on the ESO/SERC films or POSS prints measured from the lower left corner in $\mathrm{mm}$ of the field. G272.6-05.3 showed no emission lines but a continuum, therefore it probably is a galaxy.

\section{Results and discussion}

For three objects narrow band images have been obtained, the remaining images are reproductions from ESO/SERC films. In all images north is up and east to the left. A summary of the identified emission lines and some derived properties, like electron density, extinction coefficient, radial velocity is given in Table 2; for $\mathrm{NeVe} 3-1$ values for both the eastern crescent and the inner part are listed. The excitation class is derived from the ratio of the [O III] lines at 4959 and $5007 \AA$ and $\mathrm{H}_{\beta}$, follwing Aller's (1956) scheme.

KeWe 2 appeared as an elliptical ring of nebulosity on the ESO/SERC. In contrast to most elliptical PNe it shows brightness enhancements at the ends of the $m a-$ jor axis. The new narrow-band image $([\mathrm{N} \mathrm{II}])$ reveals faint outer extensions that indicate that this object is not really an elliptical but rather a bipolar PN with a pronounced equatorial density enhancement and very faint lobes; on the survey only the ring forming the waist of the butterfly according to the classification schemes of Balick (1987) and Schwarz et al. (1993) can be seen. The equatorial ring is seen almost face on. The spectrum shows a low excitation nebula with strong [ $\mathrm{N}$ II] emission. $\mathrm{H}_{\beta}$ and [O III] are very faint but from comparison with $\mathrm{H}_{\alpha}$ it is obvious that reddening must be rather small, while the ratio of $\mathrm{H}_{\beta}$ and [O III] indicates an excitation class $<4$.
Table 2. Line fluxes for the new $\mathrm{PNe}$

\begin{tabular}{|c|c|c|c|}
\hline Line $(\lambda[\AA])$ & KeWe 2 & KeWe 3 & KeWe 4 \\
\hline $\mathrm{H}_{\beta} 4861$ & 100:: & & 100 \\
\hline [O III] 4959 & & 34 & \\
\hline [O III 5007 & 170: & 100 & 1070 \\
\hline He I 5875 & & & 100 \\
\hline [O I] 6300 & & & \\
\hline [O I] 6363 & & & \\
\hline [N II] 6548 & 600 & 380 & 50 \\
\hline $\mathrm{H}_{\alpha} 6563$ & 420 & 110 & 470 \\
\hline [N II] 6584 & 1740 & 1150 & 135 \\
\hline [S II $] 6717$ & & 80: & 35 \\
\hline [S II] 6731 & & 90: & 20 \\
\hline$v_{\mathrm{rad}}\left[\mathrm{km} \mathrm{s}^{-1}\right]$ & 110 & 90 & 40 \\
\hline $\mathrm{EC}$ & $<4$ & & $6-7$ \\
\hline$c$ & $<0.5$ & & 0.7 \\
\hline$n_{\mathrm{e}}$ & - & - & $<100$ \\
\hline $\log F\left(\mathrm{H}_{\beta}\right)$ & - & - & -13.6 \\
\hline Line $(\lambda[\AA])$ & $\mathrm{NeVe} 3-1$ & NeVe 3-2 & KeWe 5 \\
\hline $\mathrm{H}_{\beta} 4861$ & & 100 & 100 \\
\hline [O III] 4959 & 40 & 175 & 280 \\
\hline [O III] 5007 & 100 & 540 & 785 \\
\hline Не I 5875 & & 30 & \\
\hline [O I] 6300 & 55 & & \\
\hline [O I] 6363 & 30 & & \\
\hline [N II] 6548 & $260 / 69$ & & 575 \\
\hline $\mathrm{H}_{\alpha} 6563$ & $340 / 100$ & 525 & 665 \\
\hline [N II] 6584 & $780 / 140$ & 11 & 1680 \\
\hline [S II] 6717 & $170 / 60$ & & 345 \\
\hline [S II] 6731 & $140 / 56$ & & 255 \\
\hline$v_{\mathrm{rad}}\left[\mathrm{km} \mathrm{s}^{-1}\right]$ & 0 & -80 & -10 \\
\hline $\mathrm{EC}$ & $4-5$ & 5 & 5 \\
\hline$c$ & 1.1 & 0.8 & 1.1 \\
\hline$n_{\mathrm{e}}$ & $150 / 450$ & 1000 & $<100$ \\
\hline $\log F\left(\mathrm{H}_{\beta}\right)$ & - & -12.5 & -14.4 \\
\hline
\end{tabular}



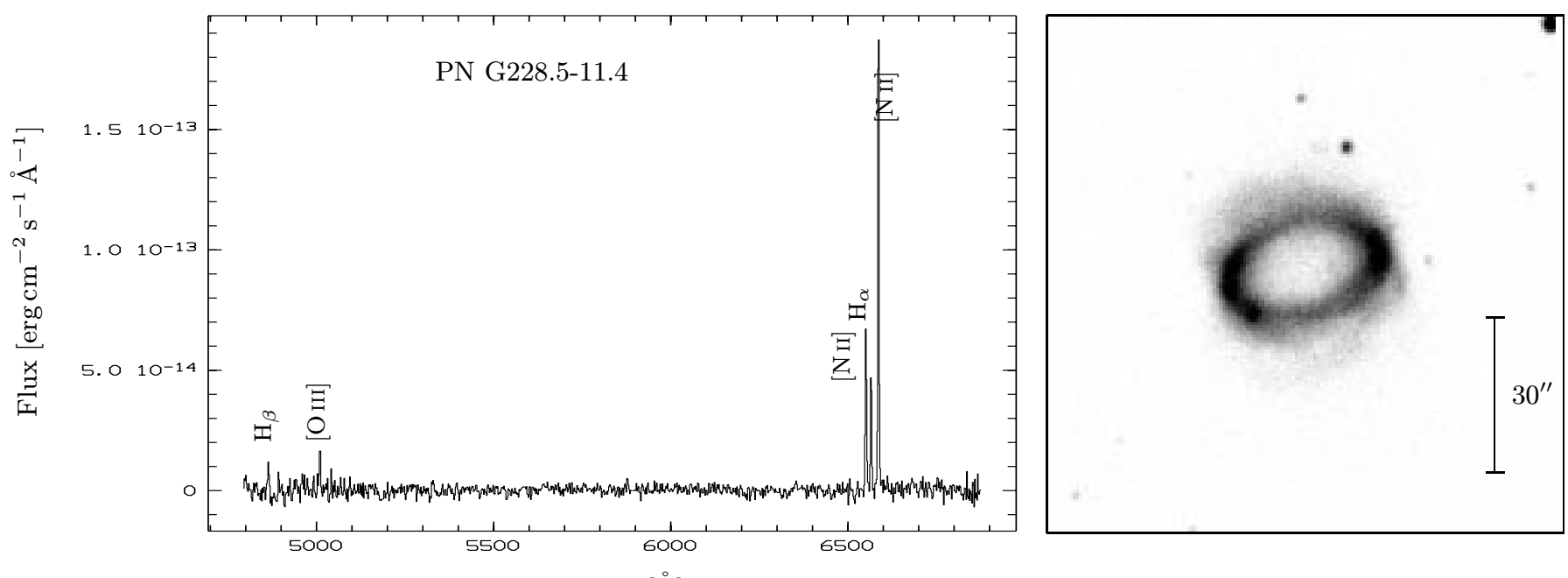

Wavelength $[\AA]$

Fig. 1. Spectrum and [NII]-image for object KeWe 2, PN G228.5-11.4
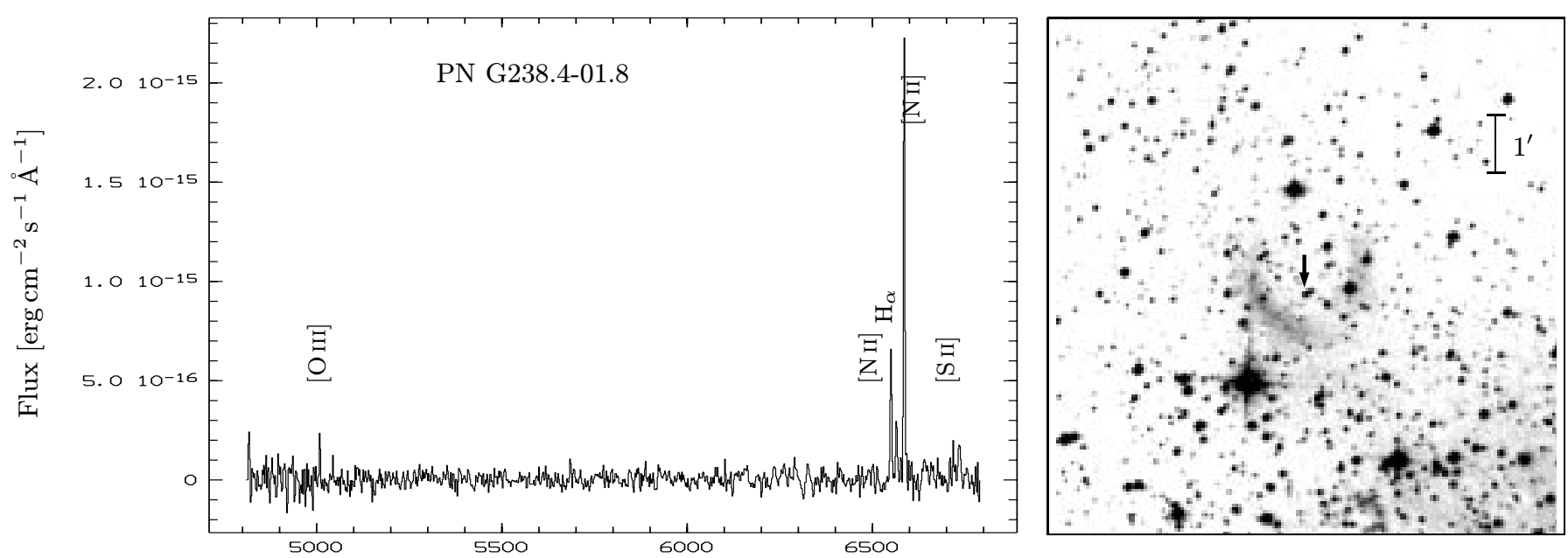

Wavelength $[\AA]$

Fig. 2. Spectrum and ESO-R-image for KeWe 3, PN G238.4-01.8
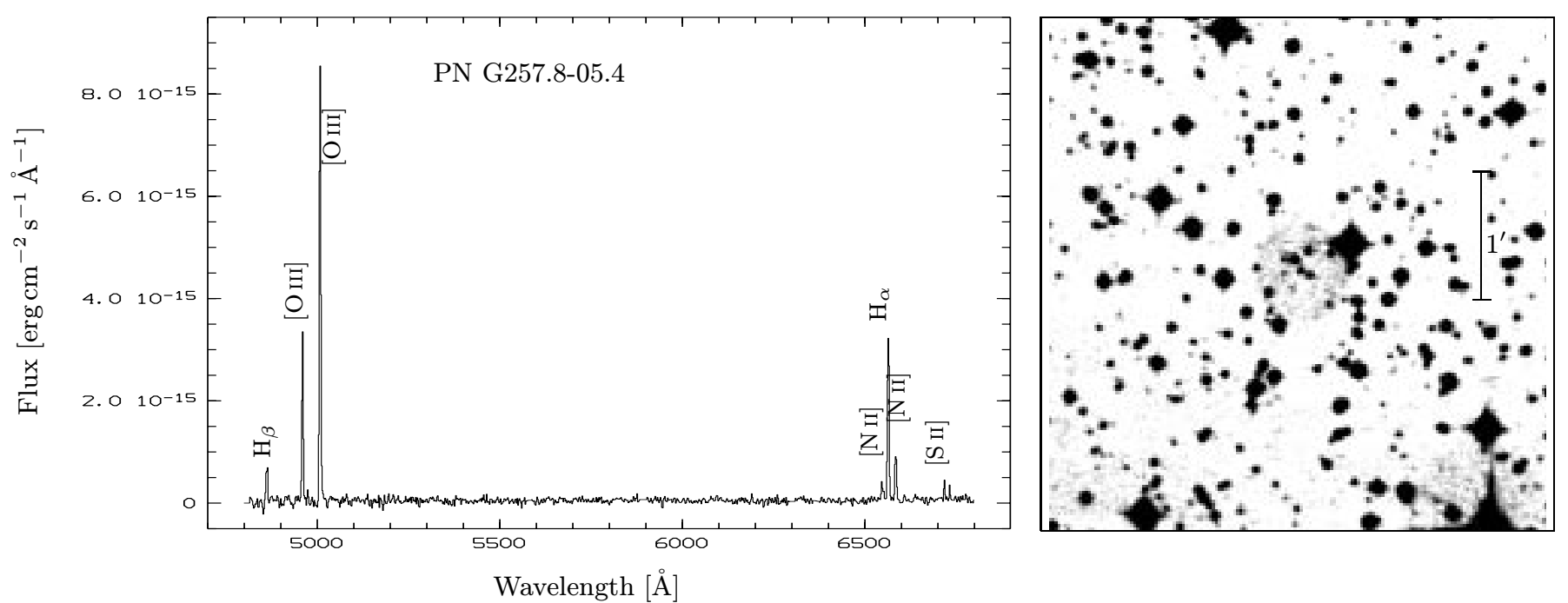

Fig. 3. Spectrum and ESO-R-image for KeWe 4, PN G257.8-05.4 

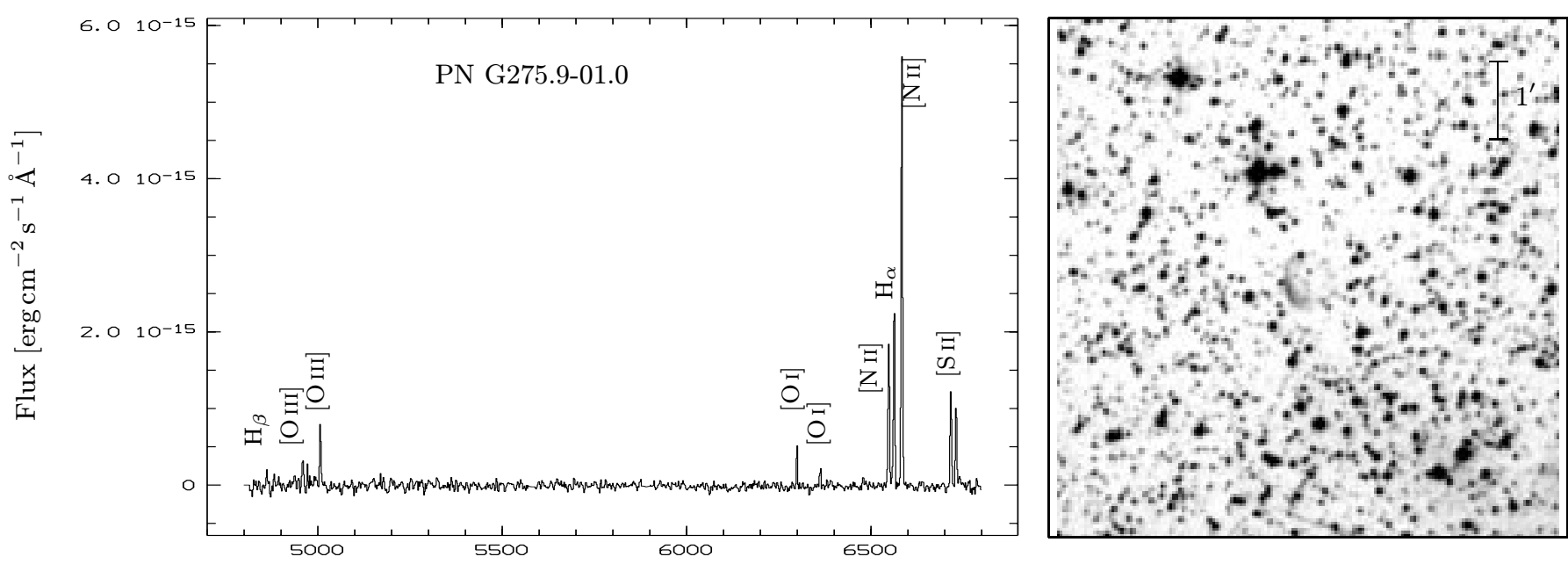

Wavelength $[\AA]$

Fig. 4. Spectrum of eastern crescent and ESO-R-image for NeVe 3-1, PN G275.9-01.0
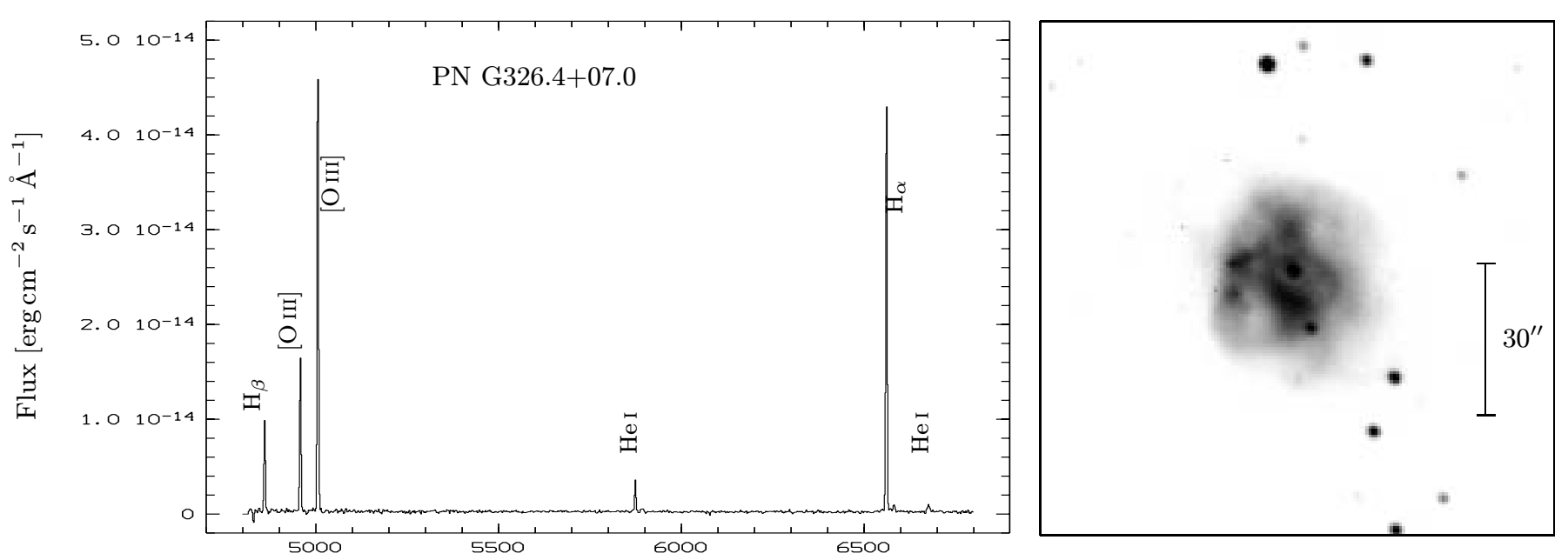

Wavelength $[\AA]$

Fig. 5. Spectrum and $\left(\mathrm{H}_{\alpha}+[\mathrm{NII}]\right)$-image for NeVe 3-2, PN G326.4+07.0
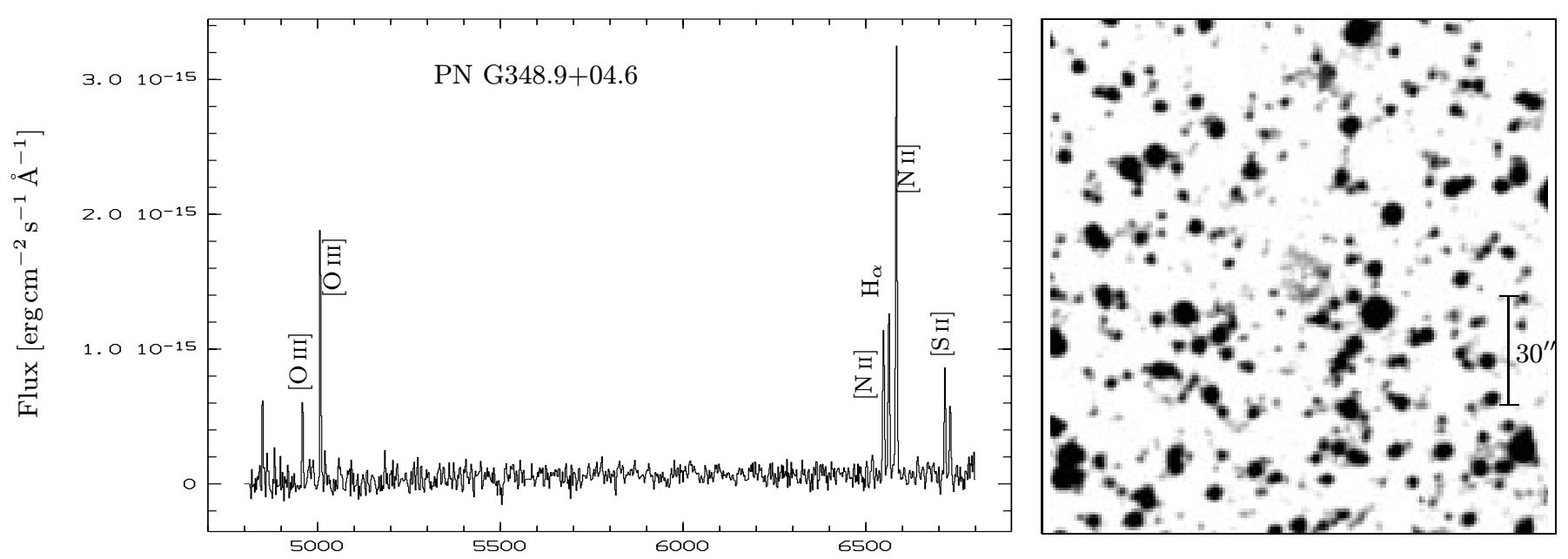

Wavelength $[\AA]$

Fig. 6. Spectrum and ESO-R-image for KeWe 5, PN G348.9+04.6 

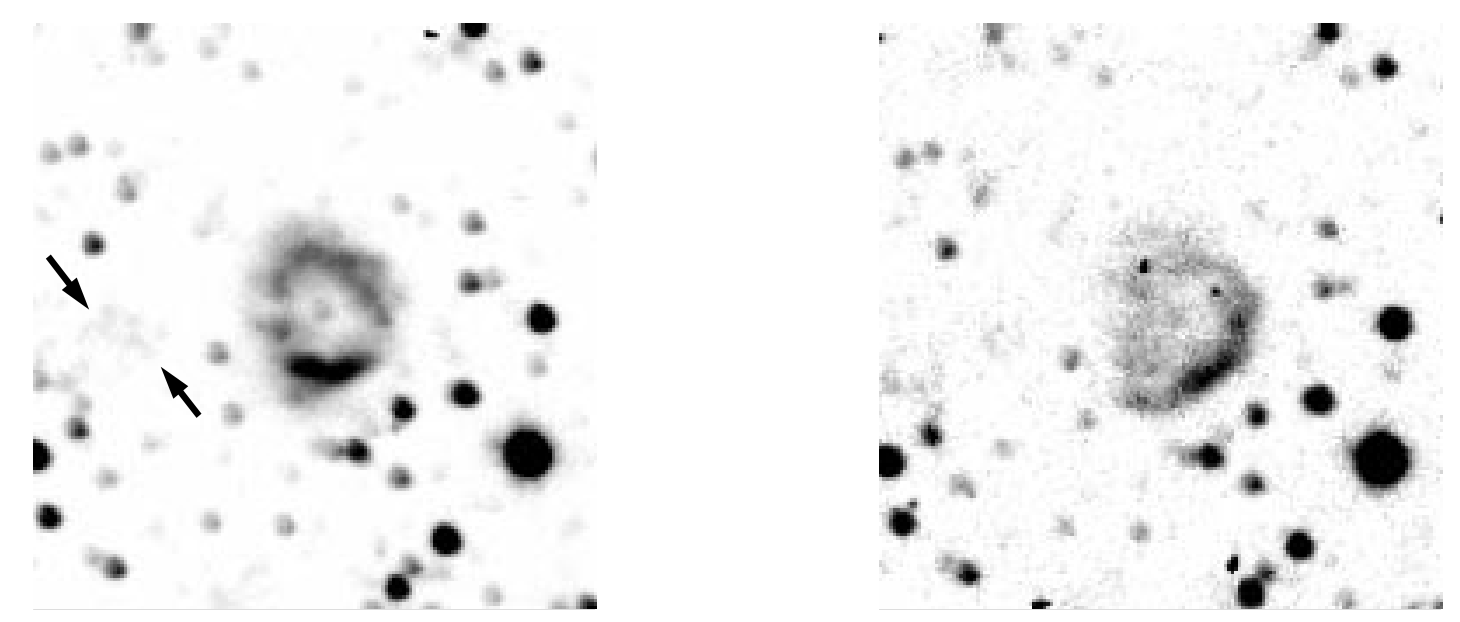

Fig. 7. $\left(\mathrm{H}_{\alpha}+[\mathrm{NII}]\right)$ and $[\mathrm{O} \mathrm{III}]$ images for $\mathrm{KeWe} 5$, PN G348.9+04.6

KeWe 3 is a rather large ( 2 arcmin) nebula consisting of two crescent shaped arcs forming a highly incomplete shell. The eastern crescent is brighter on the ESO/SERC. A possible central star is identified in Fig. $2 \mathrm{~b}$. The spectrum is remarkable showing strong $[\mathrm{N}$ II] emission but very weak lines of $\mathrm{H}_{\alpha}$ and $[\mathrm{O} \mathrm{III}]$. The strange nitrogen ring nebula PL 1547.3-5612 has a very similar spectrum (Ruiz 1983). Due to the lack of useable lines no reliable information on reddening or density of the object could be derived. Still the distinct morphology and the extreme $\mathrm{H}_{\alpha}$ to [N $\left.\mathrm{II}\right]$ ratio makes this object a likely candidate for an evolved $\mathrm{PN}$ in an advanced stage of interaction with the ISM. This is corroborated by the fact that no complete shell is visible, compared with A 34 and A 61 whose structure is still intact, see Tweedy \& Kwitter (1994).

From a morphological point of view KeWe 4 is a typical old PN - interestingly enough it is the only such archetypical object in our sample - sporting a disklike structure of homogeneous but extremely low surface brightness and a central star (CS). Similarily the physical properties derived from the spectrum are quite ordinary for an evolved PN. A comparatively small reddening of $c=0.7$ is obtained from the Balmer decrement, while the excitation class from $\mathrm{H}_{\beta}$ and $[\mathrm{O} \mathrm{II}]$ is relatively high, $\mathrm{EC} \approx 6-7$. The sulfur doublet gives an electron density of $<100 \mathrm{~cm}^{-3}$ making this a normal old $\mathrm{PN}$ at a statistical distance of $3.5 \mathrm{kpc}$ (diameter $\approx 0.7 \mathrm{pc}$ ) that would certainly have been discovered and identified much earlier had it not been for its extremely low surface brightness.

NeVe 3-1 looks somewhat similar to KeWe 5 but the brightness distribution is even more lopsided with the $\mathrm{E}$ crescent being much brighter. In the central region that appears empty on the ESO/SERC the spectrum is of poor $\mathrm{S} / \mathrm{N}$ and therefore it is not shown here, the only useful parameters derived are a density of $\approx 450$ from the $[\mathrm{S} \mathrm{II}]$ lines and a $\mathrm{H}_{\alpha}$ to [N II] ratio of 0.7. The spectrum of the two crescents are very interesting: both show a nebula of medium excitation (EC $4-5)$ with strong [N II] lines and considerable reddening, $c=1.1$. Comparison of the two spectra reveals a number of important differences, in particular some observed line ratios are not constant. While the ratio of $\mathrm{H}_{\alpha} /[\mathrm{O} \mathrm{III}]$ is essentially constant the ratios of $\mathrm{H}_{\alpha} /[\mathrm{NII}]$ and $[\mathrm{N} \mathrm{II}] /[\mathrm{O} \mathrm{III}]$ are not. [N $\left.\mathrm{NI}\right]$ is much stronger in the East, the brighter crescent. The electron density in both crescents is decidedly lower than in the central region (450); an upper limit of $<100$ is found for the fainter arc, compared to a value of $\approx 150$ in the brighter one. These observational facts: onesided brightness enhancement, enhancement of density and lower ionisation stages are exactly what is predicted for an evolved PN interacting with the ISM (Soker et al. 1991). NeVe 3-1 might be an excellent object to study these processes in more detail.

$\mathrm{NeVe} 3-2$ is a roundish nebula with a bright central object. The spectrum is dominated by the Balmer lines and [O III] emission, HeI is present, while the low ionisation stages like $[\mathrm{NII}]$ and $[\mathrm{OI}]$ are very weak. In fact almost all [N II] seems to be concentrated in a small knot east of the CS, as seen on the raw data, that has a density of $1000 \mathrm{~cm}^{-3}$. The reddening is determined as $c=0.8$ from the Balmer decrement giving a diameter of $0.25 \mathrm{pc}$ at a statistical distance of $1.8 \mathrm{kpc}$.

At first sight KeWe 5 looks like an ordinary PN on the ESO/SERC showing a circular shape and a CS. The spectrum reveals a nebula of medium excitation with strong [N II] emission that must be in a highly evolved state since the $[\mathrm{S} \mathrm{II}]$ doublet is in its low density limit, indicating a density $<100 \mathrm{~cm}^{-3}$. At a distance of some 15 arcseconds a faint nebular emission was identified in the spectrum; despite its poor $\mathrm{S} / \mathrm{N}$ ratio it is obvious that the ratio of $\mathrm{H}_{\alpha}$ to $[\mathrm{N}$ II] is higher in this patch $(\approx 0.8)$ than in the nebula itself (0.4); the location of this faint nebular patch is marked in the $\left(\mathrm{H}_{\alpha}+[\mathrm{NII}]\right)$-image by two arrows. Note that this patch cannot be explained by diffuse galactic emission which has been found in [S II] by Kingsburgh \& English (1992) around a number of objects. A statistical distance of $8 \mathrm{kpc}$ is found but this can only be considered 
a very rough estimate due to the low $\mathrm{H}_{\beta}$ flux observed. The narrow band images taken in $\mathrm{H}_{\alpha}$ and [O III] reveal a much more complicated structure than the ESO/SERC. In the $\left(\mathrm{H}_{\alpha}+[\mathrm{NII}]\right)$ image the $\mathrm{PN}$ looks like a broken elliptical ring with a very pronounced brightness enhancement in the North. In [O III] in contrast the object takes on an oddly deformed shape again showing a strong brightness enhancement but the position does not coincide with the brightness maximum in $\mathrm{H}_{\alpha}$. Concentrated asymmetric brightness enhancements and disrupted structure are suggestive of an interaction with the interstellar medium (ISM). This could also account for the strong [N II] emission, see Borkowski et al. (1990) and Tweedy \& Kwitter (1994) for details. The low density observed is also consistent with this notion.

KeWe 2, KeWe 3, NeVe 3-1 and KeWe 5 all show very high $[\mathrm{N} \mathrm{II}] / \mathrm{H}_{\alpha}$ and $[\mathrm{S} \mathrm{II}] / \mathrm{H}_{\alpha}$ ratios. In fact the observed $[\mathrm{N} \mathrm{II}] / \mathrm{H}_{\alpha}$ ratios are among the highest ever found in $\mathrm{PNe}$ (e.g. Guerrero et al. 1995). Unfortunately, due to the poor signal to noise ratio of the spectra, a proper analysis of the abundances of the ionized gas could not be carried out. However we have placed those values in a diagnostic diagram (Canto 1981), and they are located very close to the position of the supernova remants. This indicates that a strong contribution of the excitation in these nebulae is due to shock.

\section{Conclusion}

In conclusion we'd like to reiterate a point already made in Paper I: due to the extreme heterogeneity of the class each PN is, to a certain extent, unique. So every new addition is well worth studying in detail, despite the more than 1000 galactic PNe known. The new objects presented here vividly illustrate this. Of the six objects in our sample three show signs of interaction with the ISM. They rank among the smallest but also most promising examples yet identified. In fact their small angular size should make them very good targets for further study by spectroscopy and narrow-band CCD imaging despite their low surface brightness. A solid observational base combined with further theoretical study will definitely be able to help better understand this process that seems to be much more common and important in the late stages of PN evolution than once thought. Additionally it provides a unique opportunity to study the properties of the ISM.
Acknowledgements. We want to thank R. Weinberger and S. Kimeswenger for their support and fruitful discussions. Special thanks are also due to the members of the staff at Las Campanas Observatory for their assistance and hospitality. We gratefully acknowledge the financial support by the "Fonds zur Förderung der wissenschaftlichen Forschung"; project P10279-AST and a travel grant from the Austrian "Bundesministerium für Wissenschaft, Transport und Kunst". This research has made use of the Simbad database, operated at CDS, Strasbourg, France.

\section{References}

Acker A., Marcout J. Ochsenbein F., et al., 1996, First Supplement to the Strasbourg-ESO Catalogue of Galactic Planetary Nebulae

Acker A., Ochsenbein F., Stenholm B., et al., 1992, StrasbourgESO Catalogue of Galactic Planetary Nebulae

Aller L.H., 1956, Gaseous Nebulae, p. 66

Balick B., 1987, AJ 94, 671

Borkowski K.J., Sarazin C.L., Soker N., 1990, ApJ 360, 173

Cantó J., 1981, in: Investigating the Universe. Reidel, p. 95

Guerrero M.A., Manchado A., Stanghellini L., 1995, ApJ 444, L49

Hamuy M., Suntzeff N.B., Heathcote S.R., et al., 1994, PASP 106,566

Hamuy M., Walker A.R., Suntzeff N.B., et al., 1992, PASP 104, 533

Hollis J.M., van Buren D., Vogel S.N., et al., 1996, ApJ 456, 644

Jacoby G.H., 1981, ApJ 244, 903

Kerber F., Lercher G., Weinberger R., 1996, A\&AS 119, 423 (Paper I)

Kingsburgh R.L., English J., 1992, MNRAS 259, 635

Melmer D., Weinberger R., 1990, MNRAS 243, 236

Neckel Th., Vehrenberg H., 1990, Atlas of Galactic Nebulae III. Treugesell-Verlag, Düsseldorf

Ruiz M.T., 1983, ApJ 268, L 103

Schwarz H.E., Corradi R.L.M., Stanghellini L., 1993, in: IAU Symp. 155: Planetary Nebulae, Weinberger R., Acker A. (eds.). Kluwer, Dordrecht, p. 214

Smith H., 1976, MNRAS 175, 419

Soker N., Borkowski K.J., Sarazin C.L., 1991, ApJ 102, 1381

Tweedy R.W., Kwitter K.B., 1994, AJ 108, 188

Tweedy R.W., Napiwotzki R., 1994, AJ 108, 978

Xilouris K.M., Papamastorakis J., Paleologou E., Terzian Y., 1996, A\&A 310, 603 